\title{
Are there modifiable risk factors to prevent a cerebrospinal fluid leak following vestibular schwannoma surgery?
}

\author{
William R. Copeland, MD, ${ }^{1}$ Grant W. Mallory, MD, ${ }^{1}$ Brian A. Neff, MD, ${ }^{2}$ Colin L. W. Driscoll, MD, ${ }^{1,2}$ \\ and Michael J. Link, MD ${ }^{1,2}$
}

Departments of ${ }^{1}$ Neurologic Surgery and ${ }^{2}$ Otorhinolaryngology, Mayo Clinic, Rochester, Minnesota

OBJECT The following study was conducted to identify risk factors for a postoperative CSF leak after vestibular schwannoma (VS) surgery.

METHODS The authors reviewed a prospectively maintained database of all patients who had undergone resection of a VS at the Mayo Clinic between September 1999 and May 2013. Patients who developed a postoperative CSF leak within 30 days of surgery were compared with those who did not. Data collected included patient age, sex, body mass index (BMI), tumor size, tumor side, history of prior tumor treatment, operative time, surgical approach, and extent of resection. Both univariate and multivariate regression analyses were performed to evaluate all variables as risk factors of a postoperative CSF leak.

RESULTS A total of 457 patients were included in the study, with 45 patients (9.8\%) developing a postoperative CSF leak. A significant association existed between increasing BMI and a CSF leak, with those classified as overweight (BMI 25-29.9), obese (BMI 30-39.9), or morbidly obese (BMI $\geq 40$ ) having a 2.5-, 3-, and 6-fold increased risk, respectively. Patients undergoing a translabyrinthine (TL) approach experienced a higher rate of CSF leaks (OR 2.5, 95\% Cl 1.3-4.6; $p=0.005)$, as did those who had longer operative times (OR 1.04, 95\% Cl 1.02-1.07; $p=0.0006)$. The BMI, a TL approach, and operative time remained independent risk factors on multivariate modeling.

CONCLUSIONS Elevated BMI is a risk factor for the development of a postoperative CSF leak following VS surgery. Recognizing this preoperatively can allow surgeons to better counsel patients regarding the risks of surgery as well as perhaps to alter perioperative management in an attempt to decrease the likelihood of a leak. Patients undergoing a TL approach or having longer operative times are also at increased risk of developing a postoperative CSF leak.

http://thejns.org/doi/abs/10.3171/2014.10.JNS14432

KEY WORDS cerebrospinal fluid leak; vestibular schwannoma; acoustic neuroma; predictors; risk factors; oncology

$\mathrm{C}$ EREBROSPINAL fluid leaks remain a common complication following the resection of a vestibular CSF (Fig. 1).,18 Not only does a postoperative CSF leak lead to additional use of hospital resources and increased cost to the patient, but the presence of a leak may result in patient morbidity, including meningitis or even death. ${ }^{5,16}$ This study was conducted to identify risk factors for a postoperative CSF leak after VS surgery. Identifying such risk factors could help surgeons appropriately counsel patients regarding their risk profile and potentially modify perioperative management in an attempt to reduce the risk of developing a CSF leak.

\section{Methods}

Following institutional review board approval, we reviewed a prospectively maintained database of all patients who had undergone resection of a VS at our institution between September 1999 and May 2013. Patients who developed a postoperative CSF leak, either otorhinorrhea or

ABBREVIATIONS BMI = body mass index; ICP = intracranial pressure; MF = middle fossa; $R S$ = retrosigmoid; $T L=$ translabyrinthine; $V S=$ vestibular schwannoma . SUBMITTED February 25, 2014. ACCEPTED October 8, 2014.

INCLUDE WHEN CITING Published online November 21, 2014; DOI: 10.3171/2014.10.JNS14432.

DISCLOSURE The authors report no conflict of interest concerning the materials or methods used in this study or the findings specified in this paper. 


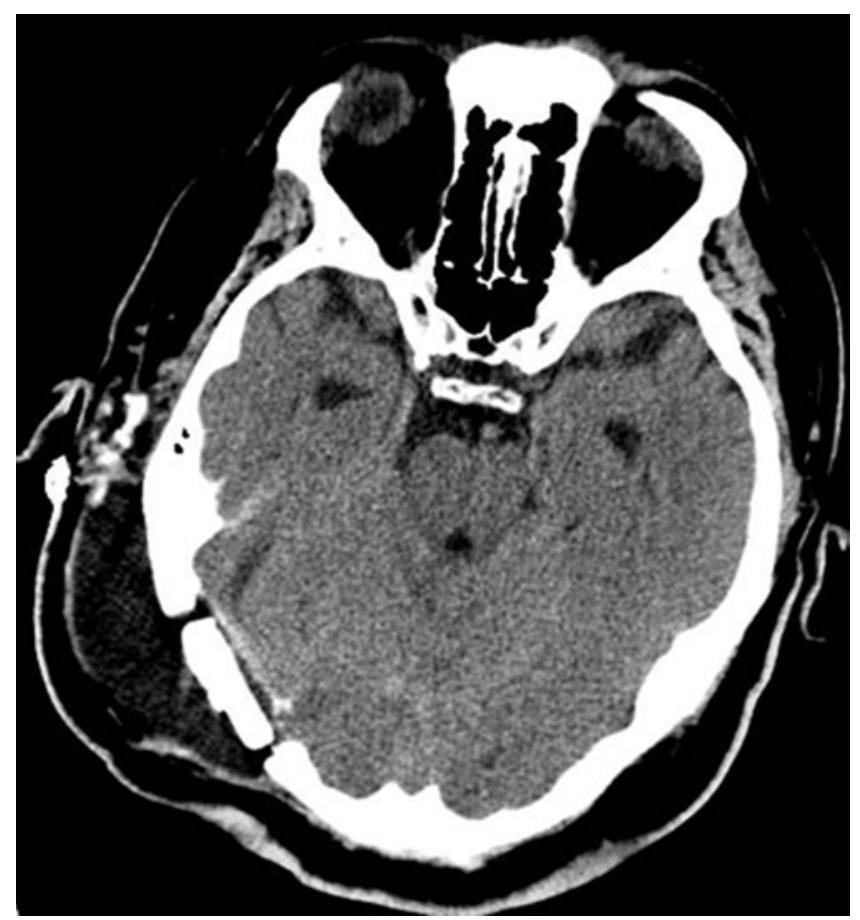

FIG. 1. Axial image from a head CT obtained without contrast, demonstrating a large pseudomeningocele in a 68-year-old man with a BMI of 42 who underwent resection of a right-sided $3.3-\mathrm{cm}$ VS via an RS approach. Postoperatively he developed CSF rhinorrhea that was treated with oversewing of his ear canal and obliteration of the middle ear. Ultimately he required a ventriculoperitoneal shunt due to symptomatic hydrocephalus.

externally from the wound, were identified. Those patients who developed a delayed leak, defined as more than 30 days after surgery, were excluded from the study. The remainder of patients who did not experience a CSF leak were used as a control group.

Variables that were possibly predictive of a postoperative CSF leak were analyzed, including the following: patient age; sex; body mass index (BMI); tumor size; tumor side; history of prior tumor treatment (surgery or radiation); operative time; surgical approach (retrosigmoid $[\mathrm{RS}]$, translabyrinthine [TL], or middle fossa [MF]); and extent of resection. Extent of resection was judged by the independent intraoperative impression of the neurosurgeon and neurootologist, and correlated to the initial 3-month follow-up MRI scan reviewed by a neuroradiologist who was blinded to the surgeon's description of the extent of resection. Resection was categorized as gross total (when no residual tumor was seen at surgery or on MRI), near total (when a tumor remnant smaller than 5 $\times 5 \times 2 \mathrm{~mm}$ was intentionally left and was not visible on MRI), or subtotal (when anything more than the remnant left in a near-total resection was left behind).

Statistical analysis was performed using JMP software. Univariate regression was used to assess for strength of association between collected variables and a postoperative CSF leak. The BMI was analyzed as both a categorical and continuous variable, whereas patient age, tumor size, and length of operative time were analyzed as continuous variables only. All other variables were categorical. Variables with a $\mathrm{p}$ value of $\leq 0.05$ on univariate testing were included in multivariate modeling.

\section{Results}

\section{Patient Demographic Data}

A total of 462 patients with VSs underwent resection over the study period. Five patients were excluded because they developed delayed CSF leaks, beginning between 10 months and 4 years after surgery. Therefore, 457 patients remained for the final analysis. The mean age of patients was 48 years (range 14-86 years), with females comprising $52 \%$ of the cohort. Patients had a mean BMI of $28 \mathrm{~kg} /$ $\mathrm{m}^{2}$ (range $12.7-50.4 \mathrm{~kg} / \mathrm{m}^{2}$ ); $37 \%$ were classified as overweight (BMI 25-29.9), 30\% were obese (BMI 30-39.9), and $4 \%$ were morbidly obese (BMI $\geq 40)$. The mean tumor diameter was $2.7 \mathrm{~cm}$ (ranging from intracanalicular to 5.5 $\mathrm{cm}$ ) as measured at the largest posterior fossa dimension. A total of 32 patients (7\%) underwent prior radiation and/ or surgery (radiation in 17, surgery in 15). The most common surgical approach was an RS approach (56\%), followed by TL (36\%) and MF (8\%) approaches. The mean operative time was 6 hours and 13 minutes (range 107-760 minutes). Extent of resection was gross total in $71 \%$ of tumors, near total in $13 \%$, and subtotal in $16 \%$.

Forty-five patients $(9.8 \%)$ developed postoperative CSF leaks within 30 days of surgery. Of these, 12 patients had leakage from the wound and 33 had otorhinorrhea. A com-

TABLE 1. Comparison of demographic data in 457 patients with and without CSF leaks after VS surgery*

\begin{tabular}{lccr}
\hline \multicolumn{1}{c}{ Characteristic } & CSF Leak & $\begin{array}{c}\text { No CSF } \\
\text { Leak }\end{array}$ & p Value \\
\hline No. of patients & 45 & 412 & \\
\hline Age in yrs & 45.7 & 48.6 & 0.19 \\
\hline Female sex & $21(47)$ & $218(53)$ & 0.41 \\
\hline Mean BMl in kg/m² & 30.6 & 28.0 & $<0.01$ \\
\hline Normal weight; BMI 18.5-24.9 & $6(13)$ & $126(31)$ & \\
\hline Overweight; BMI 25-29.9 & $18(40)$ & $153(37)$ & \\
\hline Obese; BMI 30-39.9 & $17(38)$ & $119(29)$ & \\
\hline Morbidly obese; BMI $\geq 40$ & $4(9)$ & $14(3)$ & \\
\hline Tumor size in cm & 2.68 & 2.73 & 0.86 \\
\hline Tumor on rt side & $28(62)$ & $201(49)$ & 0.09 \\
\hline Prior treatment & $6(13)$ & $26(6)$ & 0.26 \\
\hline Operative time in mins & 434 & 367 & $<0.01$ \\
\hline Approach & & & 0.02 \\
\hline RS & $18(40)$ & $240(58)$ & \\
\hline TL & $25(56)$ & $140(34)$ & \\
\hline MF & $2(4)$ & $32(8)$ & \\
\hline Extent of resection & & & \\
\hline GTR & $32(71)$ & $294(71)$ & \\
\hline NTR & $7(16)$ & $50(12)$ & \\
\hline STR & $6(13)$ & $68(17)$ & \\
\hline GTR & & & \\
\hline
\end{tabular}

GTR = gross-total resection; NTR = near-total resection; STR = subtotal resection.

* Unless otherwise specified, the values denote number of patients (\%). 
TABLE 2. Results of univariate regression analysis in patients with CSF leaks after VS surgery

\begin{tabular}{llcr}
\hline \multicolumn{1}{c}{ Variable } & OR & $95 \% \mathrm{Cl}$ & p Value \\
\hline Significant predictors & & & \\
\hline BMI & 1.06 & $1.01-1.10$ & 0.01 \\
\hline Operative time & 1.04 & $1.02-1.07$ & $<0.01$ \\
\hline TL approach & 2.5 & $1.3-4.6$ & $<0.01$ \\
\hline Other predictors & & & \\
\hline Age & 0.99 & $0.96-1.01$ & 0.19 \\
\hline Sex & 1.3 & $0.70-2.42$ & 0.41 \\
\hline Extent of resection & 1.28 & $0.50-2.91$ & 0.73 \\
\hline Tumor size & 1.42 & $0.77-3.41$ & 0.38 \\
\hline Tumor on rt side & 1.73 & $0.93-3.31$ & 0.09 \\
\hline Any prior treatment & 1.78 & $0.58-4.54$ & 0.29 \\
\hline Prior radiation & 1.23 & $0.19-4.57$ & 0.79 \\
\hline Prior surgery & 2.19 & $0.49-7.14$ & 0.23 \\
\hline
\end{tabular}

parison of demographic data between patients who developed a leak and those who did not is shown in Table 1.

\section{Regression Analysis}

Results of the univariate regression analysis are summarized in Table 2. No significant associations were found between the development of a postoperative CSF leak and patient age, sex, tumor size, prior tumor treatment, or extent of resection. There was a trend for patients with right-sided tumors to have an increased rate of CSF leaks, although this was not statistically significant (OR $1.73,95 \%$ CI 0.93 $3.31 ; p=0.09$ ). Length of operative time did significantly correlate with the development of a postoperative CSF leak; the mean operative time in those who developed a leak was 7 hours and 14 minutes compared with 6 hours and 8 minutes in those who did not (OR 1.04, 95\% CI 1.021.07; $p=0.0006$ ). A significant association was also found between CSF leaks and a TL approach, with leak rates of $15 \%$ compared with $7 \%$ among other approaches (OR 2.5, 95\% CI 1.3-4.6; $p=0.005$ ). Finally, BMI was a significant predictor of postoperative CSF leaks; those classified as overweight (BMI 25-29.9), obese (BMI 30-39.9), or morbidly obese (BMI $\geq 40$ ) had a 2.5-, 3-, and 6-fold increased risk, respectively (Table 3). Length of operative time ( $\mathrm{p}=$ $0.0007)$, a TL approach $(\mathrm{p}=0.02)$, and BMI $(\mathrm{p}=0.04)$ each remained independent predictors of a postoperative CSF leak in multivariate modeling.

\section{Discussion}

\section{Elevated BMI as a Risk Factor for a CSF Leak}

Many factors potentially contributing to the develop- ment of a postoperative CSF leak following VS surgery have been described. Whereas factors such as age, sex, and tumor size did not correlate with the occurrence of a CSF leak in our series, other studies have reported these factors to be significant., ${ }^{4,511,20}$ These factors, among others, are fixed variables and, therefore, even if believed to influence the likelihood of a postoperative CSF leak, can only be used to counsel patients with regard to their risk of this complication. The fixed nature of these variables prevents either the surgeon or the patient from altering their effect.

Body mass index, however, is not fixed but modifiable. Since 1960, the prevalence of obesity among adults in the US has tripled, now becoming a health care epidemic affecting more than one-third of the population. ${ }^{9,10}$ It has been well established that obesity is an independent risk factor for the development of surgical site infections and other systemic postoperative complications. ${ }^{1}$ Recently there has been growing evidence that obesity is also a risk factor for development of spontaneous temporal bone CSF leaks. ${ }^{6,12,17,21}$ In contrast, little has been written regarding the possible association between obesity and the occurrence of a CSF leak following VS surgery. ${ }^{8,14}$ Diaz Anadon et al. studied 170 patients who had undergone surgery for tumors in the cerebellopontine angle (163 of whom had a VS) and found no relationship between CSF leaks and BMI. On the contrary, Mantravadi et al. evaluated 134 patients (83 of whom had a VS) and concluded that BMI was a significant predictor of CSF leaks in patients undergoing removal of tumors in the cerebellopontine angle (OR 1.10, $\mathrm{p}=0.004)$. When compared with these two prior studies, our series is unique in that we reviewed a much larger cohort of 457 patients, all of whom exclusively underwent VS resection. Similar to Mantravadi et al., we too found that obesity (BMI $\geq 30$ ) increases a patient's likelihood of developing a postoperative CSF leak, with these patients having a 3-fold increased risk. Somewhat surprisingly, an increased risk even extended to those individuals who were classified as simply overweight (BMI 25-29.9); they had a 2.5-fold increased risk of CSF leakage.

The reason why an elevated BMI makes one prone to develop a CSF leak is not entirely clear. Obesity is known to adversely affect wound healing $;{ }^{14}$ however, altered wound healing alone seems unlikely to account for the increased risk of a CSF leak because only 12 of 45 patients experienced leakage through their incision. A more convincing reason is that overweight individuals may have elevated intracranial pressure (ICP) at baseline. This has been noted by several authors, particularly in patients with spontaneous CSF leaks and idiopathic intracranial hypertension. ${ }^{7,12,17,21,22}$ It has been demonstrated that intraabdominal pressure in obese patients is elevated and that this in turn translates into higher intrathoracic and cardiac filling

TABLE 3. Impact of BMI on rate of CSF leakage after VS surgery

\begin{tabular}{lcccc}
\hline \multicolumn{1}{c}{ BMI Class } & Total & No. (\%) w/ CSF Leak & OR (95\% Cl) & $p$ Value \\
\hline Normal weight; BMI 18.5-24.9 & 132 & $6(5)$ & Reference & Reference \\
\hline Overweight; BMI 25-29.9 & 171 & $18(10.5)$ & $2.5(1.0-7.0)$ & 0.04 \\
\hline Obese; BMI 30-39.9 & 136 & $17(12.5)$ & $3.0(1.2-8.5)$ & 0.02 \\
\hline Morbidly obese; BMI $\geq 40$ & 18 & $4(22)$ & $6.0(1.4-23.7)$ & 0.02 \\
\hline
\end{tabular}


pressures, thus decreasing venous return from the brain and ultimately increasing ICP. ${ }^{22}$ Some have postulated that the increased amount of estrogen produced by adipose tissue in obese patients may play a role in the development of elevated ICP - abnormally high levels of estrogen have been found in the CSF of obese patients with idiopathic intracranial hypertension. ${ }^{23}$ Finally, obstructive sleep apnea, often associated with obesity, may elevate ICP via cerebral vasodilation resulting from hypoxia and hypercapnia. ${ }^{13,15}$ The aforementioned reasons lend support and a rationale as to why patients with an elevated BMI have a higher risk of a postoperative CSF leak.

Identifying an elevated BMI as a risk factor for a postoperative CSF leak is important not only because it can allow surgeons to identify and appropriately counsel patients at increased risk preoperatively, but it also allows the opportunity to modify this risk. For what is most commonly a nonemergency, elective operation, patients could be encouraged to lose weight prior to proceeding with surgery. Weight loss could also help prevent many of the other perioperative complications known to have an increased risk in obese patients. Recognizing, however, that certain symptomatic tumors may not allow for such a strategy and that to a patient the abstract risk of a CSF leak is unlikely to be enough incentive to accomplish substantial weight loss, the treating surgeon can still use other strategies to attenuate this risk. For example, a lumbar drain could be placed at the time of surgery and continued postoperatively for a short duration in hopes of decreasing the likelihood of developing a leak. A lumbar drain may have the added benefit of aiding the surgeon in accessing what could otherwise be a tight posterior fossa in those patients with elevated baseline ICP. Additionally, when operating on an overweight patient via a TL approach (also a risk factor for a CSF leak), the surgeon could choose to oversew the external ear canal and obliterate the middle ear space. This has traditionally been a technique we have reserved for patients requiring reoperation to fix a postoperative CSF leak, but it could be used prophylactically at the time of the initial operation in those patients at increased risk. Alternatively, an RS approach could be favored in all patients with an elevated BMI, unless there is a compelling reason to operate using a TL procedure.

\section{Other Risk Factors for a CSF Leak}

We found that the TL approach had an increased risk of developing a postoperative CSF leak compared with either the RS or MF approaches. This is in keeping with findings reported by others, ${ }^{19}$ although some have not experienced any difference between approaches regarding the leak rates. ${ }^{3,4}$ Despite this difference in leak rates in our series, however, we still commonly use the TL approach. We also found a significant association between longer operative times and the development of postoperative CSF leaks. Although the reasons are not entirely clear, we speculate that this may be reflective of surgeon fatigue. In addition, a longer operative time may allow for accumulation of more blood and proteinaceous debris in the CSF, thereby inhibiting normal CSF circulation postoperatively and causing at least a transient communicating hydrocephalus. Last, although it did not reach statistical significance, we did find a trend for an increased risk of a CSF leak in patients with right-sided tumors. The reason for this is again speculative, but may be due to occult occlusion of the adjacent, typically dominant, sigmoid sinus after prolonged retraction, especially when the TL approach is used. This could result in elevated ICP and predispose the patient to development of a postoperative CSF leak.

\section{Study Limitations}

Our study is a retrospective review and is therefore prone to the biases associated with this research method. Additionally, although some authors have concluded that nuances of surgical technique, primarily with respect to closure, may in fact have the biggest impact on CSF leak rates, we chose not to discuss this in detail. 16 Although we do not doubt the importance of a meticulous closure technique in preventing a CSF leak, any meaningful comparison between techniques is limited by the fact that nuances vary so tremendously among surgeons and that the reported effectiveness of different techniques is mostly anecdotal.

\section{Conclusions}

Elevated BMI is a risk factor for the development of a postoperative CSF leak following VS surgery. Recognizing this preoperatively can allow surgeons to better counsel patients regarding the risks of surgery as well as perhaps to alter perioperative management in an attempt to decrease the likelihood of developing a leak. Patients undergoing a TL approach or having longer operative times are also at increased risk of a postoperative CSF leak.

\section{References}

1. Bamgbade OA, Rutter TW, Nafiu OO, Dorje P: Postoperative complications in obese and nonobese patients. World J Surg 31:556-561, 2007

2. Bani A, Gilsbach JM: Incidence of cerebrospinal fluid leak after microsurgical removal of vestibular schwannomas. Acta Neurochir (Wien) 144:979-982, 2002

3. Becker SS, Jackler RK, Pitts LH: Cerebrospinal fluid leak after acoustic neuroma surgery: a comparison of the translabyrinthine, middle fossa, and retrosigmoid approaches. Otol Neurotol 24:107-112, 2003

4. Brennan JW, Rowed DW, Nedzelski JM, Chen JM: Cerebrospinal fluid leak after acoustic neuroma surgery: influence of tumor size and surgical approach on incidence and response to treatment. J Neurosurg 94:217-223, 2001

5. Bryce GE, Nedzelski JM, Rowed DW, Rappaport JM: Cerebrospinal fluid leaks and meningitis in acoustic neuroma surgery. Otolaryngol Head Neck Surg 104:81-87, 1991

6. Carlson ML, Copeland WR III, Driscoll CL, Link MJ, Haynes DS, Thompson RC, et al: Temporal bone encephalocele and cerebrospinal fluid fistula repair utilizing the middle cranial fossa or combined mastoid-middle cranial fossa approach. Clinical article. J Neurosurg 119:1314-1322, 2013

7. Daniels AB, Liu GT, Volpe NJ, Galetta SL, Moster ML, Newman NJ, et al: Profiles of obesity, weight gain, and quality of life in idiopathic intracranial hypertension (pseudotumor cerebri). Am J Ophthalmol 143:635-641, 2007

8. Diaz Anadon A, Lassaletta L, Roda JM, Gavilán Bouzas J: [CSF fistulae following surgery for cerebellopontine angle tumours and their relationship with the body mass index.] Acta Otorrinolaringol Esp 60:318-324, 2009 (Span) 
9. Flegal KM, Carroll MD, Kuczmarski RJ, Johnson CL: Overweight and obesity in the United States: prevalence and trends, 1960-1994. Int J Obes Relat Metab Disord 22:3947, 1998

10. Flegal KM, Carroll MD, Ogden CL, Curtin LR: Prevalence and trends in obesity among US adults, 1999-2008. JAMA 303:235-241, 2010

11. Glasscock ME III, Hays JW, Murphy JP: Complications in acoustic neuroma surgery. Ann Otol Rhinol Laryngol 84: $530-540,1975$

12. Goddard JC, Meyer T, Nguyen S, Lambert PR: New considerations in the cause of spontaneous cerebrospinal fluid otorrhea. Otol Neurotol 31:940-945, 2010

13. Jennum P, Børgesen SE: Intracranial pressure and obstructive sleep apnea. Chest 95:279-283, 1989

14. Mantravadi AV, Leonetti JP, Burgette R, Pontikis G, Marzo $\mathrm{SJ}$, Anderson D: Body mass index predicts risk for complications from transtemporal cerebellopontine angle surgery. Otolaryngol Head Neck Surg 148:460-465, 2013

15. Purvin VA, Kawasaki A, Yee RD: Papilledema and obstructive sleep apnea syndrome. Arch Ophthalmol 118:16261630,2000

16. Rodgers GK, Luxford WM: Factors affecting the development of cerebrospinal fluid leak and meningitis after translabyrinthine acoustic tumor surgery. Laryngoscope 103:959-962, 1993

17. Scurry WC Jr, Ort SA, Peterson WM, Sheehan JM, Isaacson JE: Idiopathic temporal bone encephaloceles in the obese patient. Otolaryngol Head Neck Surg 136:961-965, 2007

18. Selesnick SH, Liu JC, Jen A, Newman J: The incidence of cerebrospinal fluid leak after vestibular schwannoma surgery. Otol Neurotol 25:387-393, 2004

19. Shea MC, Robertson JT: Acoustic neuroma removal: a comparative study of translabyrinthine and suboccipital approaches. Am J Otol 1:94-99, 1979

20. Stieglitz LH, Wrede KH, Gharabaghi A, Gerganov VM, Samii A, Samii M, et al: Factors affecting postoperative cerebrospinal fluid leaks after retrosigmoidal craniotomy for vestibular schwannomas. Clinical article. J Neurosurg 111:874-883, 2009

21. Stucken EZ, Selesnick SH, Brown KD: The role of obesity in spontaneous temporal bone encephaloceles and CSF leak. Otol Neurotol 33:1412-1417, 2012

22. Sugerman HJ, DeMaria EJ, Felton WL III, Nakatsuka M, Sismanis A: Increased intra-abdominal pressure and cardiac filling pressures in obesity-associated pseudotumor cerebri. Neurology 49:507-511, 1997

23. Toscano V, Sancesario G, Bianchi P, Cicardi C, Casilli D, Giacomini P: Cerebrospinal fluid estrone in pseudotumor cerebri: a change in cerebral steroid hormone metabolism? J Endocrinol Invest 14:81-86, 1991

\section{Author Contributions}

Conception and design: Link, Copeland, Mallory. Acquisition of data: Copeland, Mallory. Analysis and interpretation of data: Copeland, Mallory. Drafting the article: Copeland, Mallory. Critically revising the article: Link, Copeland, Mallory. Reviewed submitted version of manuscript: all authors. Approved the final version of the manuscript on behalf of all authors: Link. Statistical analysis: Copeland, Mallory. Study supervision: Link, Neff, Driscoll.

\section{Supplemental Information \\ Previous Presentation}

Portions of this work were presented in abstract form at the North American Skull Base Society annual meeting, which was held in San Diego, CA, February 14-16, 2014.

\section{Correspondence}

Michael J. Link, Department of Neurological Surgery, Mayo Clinic, 200 First St. SW, Rochester, MN 55905. email: link. michael@mayo.edu. 